Journal of Computer Science 7 (12): 1894-1899, 2011

ISSN 1549-3636

(C) 2011 Science Publications

\title{
Field Programmable Gate Arrays based Design, Implementation and Delay Study of Braun's Multipliers
}

\author{
Muhammad H. Rais and Mohammed H. Al Mijalli \\ Department of Biomedical Technology,College of Applied Medical Sciences, \\ King Saud University, Riyadh, Saudi Arabia
}

\begin{abstract}
Problem statement: Parallel array multipliers are required to achieve high execution speed for Digital Signal Processing (DSP) applications. Approach: The purpose of this article is to investigate Field Programmable Gate Arrays (FPGAs) implementation of standard Braun's multipliers on Spartan-3AN, Virtex-2, Virtex-4 and Virtex-5 FPGAs using Very high speed integrated circuit Hardware Description Language (VHDL). The delay study was analyzed using Analysis Of Variance (ANOVA) method using the software Statistical Package for Social Science (SPSS) with a 0.05 confidence level was used to compare the FPGA devices. Results: The FPGA resource utilization by Virtex-5 is the lowest in value for $4 \times 4,6 \times 6,8 \times 8$ and $12 \times 12$-bit Braun's multipliers as compared to Spartan-3AN, Virtex-2 and Virtex-4 FPGAs. The average connection delays in Virtex-2 shows consistency and gradual increase in value as the size of multiplier increased. Virtex-2 FPGA demonstrates lower average connection delays as compared to Spartan3 AN, Virtex-4 and Virtex-5 FPGAs. For the maximum pin delay same observations are obtained for Virtex-2 FPGA. The anomalies in maximum pin delay and average connection delay are observed in Virtex-5, Virtex-4 and Spartan-3AN FPGAs. FPGA devices also demonstrate that as the size of multipliers increases their mean latency value is also increases. Conclusion: The FPGA resource utilization by Virtex- 5 is the lowest in value for $4 \times 4,6 \times 6,8 \times 8$ and $12 \times 12$-bit Braun's multipliers as compared to Spartan-3AN, Virtex-2 and Virtex-4 FPGAs. Even value obtained for Virtex-5 FPGA for $4 \times 4$ bit standard Braun's multiplier for number of occupied slices and look up tables are lower in value than reported in literature.
\end{abstract}

Key words: Application Specific Integrated Circuits (ASICs), Field Programmable Gate Arrays (FPGAs), Spartan-3AN, Digital Signal Processing (DSP), General Purpose Signal Processor (GPSP)

\section{INTRODUCTION}

Computational complexities of algorithms require fast and efficient parallel multipliers for Digital Signal Processing (DSP) applications. Fast Fourier Transform (FFT), Discrete Cosine Transform (DCT) and Inverse Discrete Cosine Transform (IDCT) involve multiplication intensive algorithms commonly implemented by DSP hardware. Implementation of DSP applications require the algorithms should be verified and optimized before realization. Multiplication has always been hardware- time-and power-consuming computation in airthematic operations. In many cases implementation of DSP algorithm demands using Application Specific Integrated Circuits (ASICs). This is especially Corresponding Author: Muhammad H. Rais, Department of Biomedical Technology, College of Applied Medical Sciences, King Saud University, Riyadh, Saudi Arabia required for image processing applications. Since development costs for ASICs are high, algorithms should be verified and optimized before implementation. Field Programmable Gate Arrays (FPGAs)can provide such optimization and implementation of algorithms in real time frame.

A number of research efforts have been reported for making low power multipliers (Al Mijalli, 2011a; 2011b; Rais, 2009a; 2009b; Rais, 2010a; 2010b; Rais et al., 2010; Rais and Al Mijalli, 2011a; 2011b; 2011c).

The objective of this study is to present study of standard Braun's multipliers (Yeo and Roy, 2005) using Spartan-3AN, Virtex-2, Virtex-4 and Virtex-5 FPGA devices. And also to present the statistical evaluation effect of the Braun's multipliers delays in FPGA devices using Analysis Of Variance (ANOVA) and post hoc 
Tukey's test using the Statistical Package for Social Science (SPSS).

\section{MATERIALS AND METHODS}

Architecture platform: FPGAs especially find applications in algorithms that can make use of the massive parallelism offered by their architecture. Significant speedup in computation time can be achieved by assigning computation intensive tasks to hardware and by exploiting the parallelism in algorithms. FPGAs enable a high degree of parallelism and can achieve orders of magnitude speedup over General Purpose Processors (GPPs). This is a result of increasing embedded resources available on FPGA. The inherent parallelism of the logic resources on an FPGA allows for considerable computational throughput even at a low MHz clock rates. The flexibility of the FPGA allows for even higher performance by trading off precision and range in the number format for an increased number of parallel arithmetic units. This has driven a new type of processing called reconfigurable computing, where time intensive tasks are offloaded from software to FPGAs.

Spartan-3 FPGAs: The Spartan-3 FPGA (Xilinx, 2009) is specifically designed to meet the needs of high volume, low unit cost electronic systems. The family consists of eight member offering densities ranging from 50,000 to five million system gates. The Spartan-3 FPGA consists of five fundamental programmable functional elements: CLBs, IOBs, Block RAMs, dedicated multipliers $(18 \times 18)$ and Digital Clock Managers (DCMs). Spartan-3 family includes Spartan-3L, Spartan-3E, Spartan-3A, Spartan-3A DSP, Spartan-3AN and the extended Spartan-3A FPGAs. Particularly, the Spartan$3 \mathrm{AN}$ is used as a target technology in this study.

Virtex-2 FPGAs: The Virtex-2 FPGA family Xilinx, 2007, is a platform developed for high performance from low-density to high-density designs that are based on IP cores and customized modules. The family delivers complete solutions for telecommunication, wireless, networking, video and DSP applications, including PCI, LVDS and Double-Data-Rate (DDR) interfaces. The leading-edge $0.15-0.12 \mu \mathrm{m}$ CMOS 8layer metal process and the Virtex-2 architecture are optimized for high speed with low power consumption. Combining a wide variety of flexible features and a large range of densities up to 10 million system gates, the Virtex-2 family enhances programmable logic design capabilities and is a powerful alternative to mask-programmed gates arrays. The Virtex-2 family comprises 11 members, ranging from $40 \mathrm{~K}-8 \mathrm{M}$ system gates. The Virtex-2 architecture is optimized for highdensity and high-performance logic designs.

The Virtex-2 FPGA family consists of four major elements such as Configurable Logic Blocks (CLBs), Block Select RAM, 18 $\times 18$-bit dedicated multipliers and Digital Clock Manager (DCM).

Virtex-4 FPGAs: Viretx-4 FPGAs Xilinx, 2007 consists of three platform families i.e., LX, SX and FX. Virtex-4 devices consumes approximately 50\% the power of respective Virtex-2 Pro devices due to static and dynamic power reduction enabled by triple-oxide technology and reduced core voltage and capacitance respectively. The Virtex-4 FPGA family comprises of CLBs, Block RAMs, Xtreme DSP Slices and DCMs.

Virtex-5 FPGAs: The Virtex-5 FPGA devices Xilinx, 2007 are a programmable alternative to custom ASIC technology. Virtex-5 family provides power-optimized high speed serial transceiver blocks for enhanced serial connectivity, tri-mode Ethernet MACs and highperformance PPC 440 microprocessor embedded blocks. Virtex-5 devices also use triple-oxide technology for reducing the static power consumption. Their $1.0 \mathrm{~V}$ core voltage and $65 \mathrm{~nm}$ implementation process leads also to dynamic power consumption reduction as compared to Virtex-4 devices. Advanced DSP48E slices are available in Virtex-5 FPGAs that helps in accelerating computation intensive DSP and image processing algorithms.

Braun's multipliers: Braun's multiplier is an $\mathrm{m} \times \mathrm{n}$ bit parallel multiplier and generally known as carry save multiplier and is constructed with $m \times(n-1)$ adders and $\mathrm{m} \times \mathrm{n}$ and gates. The Braun's multiplier has a glitching problem which is due to the ripple carry adder in the last stage of the multiplier.

Mathematical basis of braun's multiplier: Consider a generic $m$ by $n$ multiplication of two unsigned $n$-bit numbers $\mathrm{Y}=\mathrm{Y}_{\mathrm{m}-1} \ldots . \mathrm{Y}_{0}$ and $\mathrm{X}=\mathrm{X}_{\mathrm{n}-1 \ldots} \mathrm{X}_{0}$ :

$$
\mathrm{Y}=\sum_{\mathrm{i}=0}^{\mathrm{m}-1} \mathrm{Y}_{\mathrm{i}} 2^{\mathrm{i}}
$$




$$
X=\sum_{i=0}^{n-1} X_{i} 2^{i}
$$

The product $\mathrm{P}=\mathrm{P}_{2 \mathrm{n}-1} \ldots \mathrm{P}_{1} \mathrm{P}_{0}$, which results from multiplying the multiplicand $\mathrm{Y}$ by the multiplier $\mathrm{X}$, can be written as follows:

$$
P=\sum_{i=0}^{m-1} \sum_{j=0}^{n-1}\left(Y_{i} X_{j}\right) 2^{i+j}
$$

\section{RESULTS}

FPGA design and implementation and one way ANOVA statistics: The design of standard Braun's multipliers are done using VHDL and implemented in a Xilinx Spartan-3AN XC3S700AN (package: fgg484, speed grade: -5), Virtex-2 XC2V40 (package: fg256, speed grade: -6), Virtex-4 XC4VLX40 (package: ff668, speed grade: -12) and Virtex-5 XC5VLX50 (package: Ff676, speed grade: -3) FPGAs using the Xilinx ISE 9.2i design tool Xilinx, 2007. A one-way ANOVA is applied to compare the mean delay time for four devices, Spartan-3AN, Virtex-2, Virtex-4 and Virtex-5. The delay time for these devices is calculated using four multipliers $4 \times 4,6 \times 6,8 \times 8$ and $12 \times 12$. The statistical analysis is done by using SPSS program.

\section{DISCUSSION}

Table 1 summarizes the statistics of delay in Braun's multipliers for Spartan-3AN, Virtex-2, Virtex4 and Virtex-5 FPGAs. The lowest mean value is obtained for $4 \times 4$ bit multiplier for Virtex -5 is $6.58 \mathrm{~ns}$ as compared to Virtex-2, Virtex-4 and Spartan-3AN are 7.84, 9.5 and 12.06 ns respectively. The Virtex-5 FPGA also demonstrates lowest mean values for $6 \times 6,8 \times 8$ and $12 \times 12$ bit Braun's multipliers.

Tables 2-5 summarize the Spartan-3AN, Virtex-2, Virtex-4 and Virtex-5 FPGAs devices resources utilization for standard $4 \times 4,6 \times 6,8 \times 8$ and $12 \times 12$-bit Braun's multipliers. The FPGA resource utilization by Virtex-5 are the lowest in value for $4 \times 4,6 \times 6,8 \times 8$ and $12 \times 12$-bit Braun's multipliers as compared to Spartan$3 A N$, Virtex-2 and Virtex-4 FPGAs. Even value obtained for Virtex-5 FPGA for $4 \times 4$ bit standard Braun's multiplier for number of occupied slices and look up tables are lower in value than reported in (Anitha and Bagyaveereswaran, 2011).

Spartan-3AN, Virtex-2, Virtex-4 and Virtex-5 FPGA devices demonstrate that as the size of multipliers increases their mean latency value is also increases.

\begin{tabular}{lllll}
\multicolumn{5}{c}{ Table1: Statistics of latency in Braun's multipliers for FPGAs } \\
\hline FPGA & Bit & Mean & Std. & Std. error \\
devices & width & $(\mathrm{ns})$ & deviation $(\mathrm{ns})$ & $\begin{array}{l}\text { of mean }(\mathrm{ns}) \\
\text { of }\end{array}$ \\
\hline Spartan-3AN & $4 \times 4$ & 12.06 & 0.7162 & 0.3203 \\
& $6 \times 6$ & 13.46 & 0.5177 & 0.2315 \\
& $8 \times 8$ & 17.22 & 0.9365 & 0.4188 \\
& $12 \times 12$ & 19.84 & 1.2837 & 0.5741 \\
Virtex-2 & $4 \times 4$ & 7.84 & 0.4278 & 0.1913 \\
& $6 \times 6$ & 10.36 & 0.9659 & 0.4320 \\
& $8 \times 8$ & 12.50 & 0.7906 & 0.3536 \\
Virtex-4 & $12 \times 12$ & 16.36 & 0.2702 & 0.1208 \\
& $4 \times 4$ & 9.50 & 0.5788 & 0.2588 \\
& $6 \times 6$ & 12.06 & 0.4278 & 0.1913 \\
& $8 \times 8$ & 11.74 & 0.2191 & 0.0980 \\
Virtex-5 & $12 \times 12$ & 14.38 & 0.7727 & 0.3455 \\
& $4 \times 4$ & 6.58 & 0.2588 & 0.1158 \\
& $6 \times 6$ & 8.14 & 0.2881 & 0.1288 \\
& $8 \times 8$ & 8.60 & 0.1581 & 0.0707 \\
& $12 \times 12$ & 12.78 & 0.1304 & 0.0583 \\
& & & &
\end{tabular}

The same finding is obtained here as reported in Al Mijalli (2011a and 2011b); Rais and Al Mijalli (2011c) that as the size of multiplier increases the mean delay time also increases.

The one-way ANOVA on Spartan-3AN, Virtex-2, Virtex-4 and Virtex-5 FPGAs are shown in Table 6. There is a statistically significant difference at the 0.05 level in delay time for the four devices $[\mathrm{F}(3,76)=$ $19.546, \mathrm{p}=0.000]$ compared by using ANOVA and post-hoc Tukey HSD multiple comparison tests at the 0.05 significance level. The test indicates that the mean of delay time for Virtex-5 (Mean $=9.03$, Standard Deviation $=2.36$ ) is significantly different from the other three devices; Spartan-3AN (Mean $=15.65$, Standard Deviation $=3.26)$, Virtex-2 $($ Mean $=11.77$, Standard Deviation = 3.26) and, Virtex-4 (Mean = 11.92, Standard Deviation $=1.84$ ). However, there is no statistically significant difference in mean delay times of the devices between Virtex- 2 and Virtex-4.

Average connection delays are much lower in Virtex-2 FPGA as compared to Spartan-3AN, Virtex-4 and Virtex-5 FPGAs. As well as there is consistency and gradual increase is seen in Virtex-2 average connection delay. For the maximum pin delay same observation is obtained for Virtex-2 FPGA that the values are gradually increasing as the size of multiplier increases. The anomalies in maximum pin delay and average connection delay are observed in Virtex-5, Virtex-4 and Spartan-3AN FPGAs.

Figure 1 shows the average value of mean delay time for Spartan-3AN, Virtex-2, Virtex-4 and Virtex-5 FPGAs devices for $4 \times 4,6 \times 6,8 \times 8$ and $12 \times 12$ Braun's multipliers, which clearly indicate that the average value of mean delay time for Virtex-5 is much lower in value than Spartan-3AN, Virtex-4 and Virtex-2 FPGAs. 
J. Computer Sci., 7 (12): 1894-1899, 2011

Table 2: FPGA resource utilization for standard braun's multipliers for spartan-3AN XC3S700AN (package: fgg484, speed grade:-5) (Rais and Al Mijalli, 2011c)

\begin{tabular}{|c|c|c|c|c|c|c|c|}
\hline Bit width & Multipliers & $\begin{array}{l}\text { Four input } \\
\text { LUTs } \\
(11776)\end{array}$ & $\begin{array}{l}\text { Occupied slices } \\
(5888)\end{array}$ & $\begin{array}{l}\text { Bonded IOBs } \\
(372)\end{array}$ & $\begin{array}{l}\text { Total } \\
\text { equivalent } \\
\text { gate count }\end{array}$ & $\begin{array}{l}\text { Average } \\
\text { connection } \\
\text { delay }(\mathrm{ns})\end{array}$ & $\begin{array}{l}\text { Maximum } \\
\text { pin delay (ns) }\end{array}$ \\
\hline $\mathrm{n}=4$ & Standard & 32 & 17 & 16 & 192 & 1.336 & 3.425 \\
\hline $\mathrm{n}=6$ & Standard & 75 & 40 & 24 & 450 & 1.007 & 2.835 \\
\hline $\mathrm{n}=8$ & Standard & 133 & 69 & 32 & 798 & 1.325 & 4.187 \\
\hline $\mathrm{n}=12$ & Standard & 295 & 152 & 48 & 1770 & 1.020 & 4.626 \\
\hline
\end{tabular}

Table 3: FPGA resource utilization for standard braun's multipliers for virtex-2 XC2V40 (package: fg256, speed grade: -6)

\begin{tabular}{|c|c|c|c|c|c|c|c|}
\hline Bit width & Multipliers & $\begin{array}{l}\text { Four input } \\
\text { LUTs } \\
(512)\end{array}$ & $\begin{array}{l}\text { Occupied slices } \\
(256)\end{array}$ & $\begin{array}{l}\text { Bonded IOBs } \\
(88)\end{array}$ & $\begin{array}{l}\text { Total } \\
\text { equivalent } \\
\text { gate count }\end{array}$ & $\begin{array}{l}\text { Average } \\
\text { connection } \\
\text { delay }(\mathrm{ns})\end{array}$ & $\begin{array}{l}\text { Maximum } \\
\text { pin delay (ns) }\end{array}$ \\
\hline $\mathrm{n}=4$ & Standard & 32 & 17 & 16 & 192 & 0.489 & 1.368 \\
\hline $\mathrm{n}=6$ & Standard & 75 & 40 & 24 & 450 & 0.592 & 1.646 \\
\hline $\mathrm{n}=8$ & Standard & 133 & 69 & 32 & 798 & 0.713 & 2.116 \\
\hline $\mathrm{n}=12$ & Standard & 295 & 152 & 48 & 1770 & 0.795 & 2.450 \\
\hline
\end{tabular}

Table 4: FPGA resource utilization for standard braun's multipliers for Virtex-4 XC4LX40 (package: ff668, speed grade: -12)

\begin{tabular}{|c|c|c|c|c|c|c|c|}
\hline Bit width & Multipliers & $\begin{array}{l}\text { Four input } \\
\text { LUTs } \\
(36864)\end{array}$ & $\begin{array}{l}\text { Occupied slices } \\
(18432)\end{array}$ & $\begin{array}{l}\text { Bonded IOBs } \\
(448)\end{array}$ & $\begin{array}{l}\text { Total } \\
\text { equivalent } \\
\text { gate count }\end{array}$ & $\begin{array}{l}\text { Average } \\
\text { connection } \\
\text { delay }(\mathrm{ns})\end{array}$ & $\begin{array}{l}\text { Maximum } \\
\text { pin delay (ns) }\end{array}$ \\
\hline $\mathrm{n}=4$ & Standard & 32 & 17 & 16 & 192 & 1.037 & 2.754 \\
\hline $\mathrm{n}=6$ & Standard & 75 & 40 & 24 & 450 & 0.924 & 2.245 \\
\hline $\mathrm{n}=8$ & Standard & 133 & 70 & 32 & 798 & 0.923 & 2.333 \\
\hline $\mathrm{n}=12$ & Standard & 295 & 152 & 48 & 1770 & 0.957 & 3.011 \\
\hline
\end{tabular}

Table 5: FPGA resource utilization for standard braun's multipliers for Virtex-5 XC5VLX50 (package: ff676, speed grade:-3)

\begin{tabular}{|c|c|c|c|c|c|c|c|}
\hline Bit width & Multipliers & $\begin{array}{l}\text { Four Input } \\
\text { LUTs } \\
(28800) \\
\end{array}$ & $\begin{array}{l}\text { Occupied slices } \\
(7200)\end{array}$ & $\begin{array}{l}\text { Bonded IOBs } \\
(440)\end{array}$ & $\begin{array}{l}\text { Total } \\
\text { equivalent } \\
\text { gate count }\end{array}$ & $\begin{array}{l}\text { Average } \\
\text { connection } \\
\text { delay }(n s) \\
\end{array}$ & $\begin{array}{l}\text { Maximum } \\
\text { pin delay (ns) }\end{array}$ \\
\hline $\mathrm{n}=4$ & Standard & 22 & 11 & 16 & 154 & 0.887 & 2.103 \\
\hline $\mathrm{n}=6$ & Standard & 43 & 19 & 24 & 301 & 0.885 & 1.795 \\
\hline $\mathrm{n}=8$ & Standard & 81 & 29 & 32 & 567 & 0.857 & 1.733 \\
\hline $\mathrm{n}=12$ & Standard & 202 & 96 & 48 & 1414 & 1.074 & 2.834 \\
\hline
\end{tabular}

Table 6: The results of multiple comparisons of delay time (ns) for four devices using the Tukey's HSD post-hoc test

\begin{tabular}{|c|c|c|c|c|c|c|}
\hline \multirow[b]{2}{*}{ (I) Devices } & \multirow[b]{2}{*}{ (J) Devices } & \multirow[b]{2}{*}{ Mean difference (I-J) } & \multirow[b]{2}{*}{ Std. Error } & \multirow[b]{2}{*}{ Sig } & \multicolumn{2}{|c|}{$95 \%$ Confidence interval } \\
\hline & & & & & Lower bound & Upper bound \\
\hline \multirow[t]{3}{*}{ Spartan-3AN } & Virtex-2 & $3.88000^{*}$ & 0.86952 & 0.000 & 1.596 & 6.164 \\
\hline & Virtex-4 & $3.72500^{*}$ & 0.86952 & 0.000 & 1.441 & 6.009 \\
\hline & Virtex-5 & $6.62000^{*}$ & 0.86952 & 0.000 & 4.336 & 8.904 \\
\hline \multirow[t]{3}{*}{ Virtex-2 } & Spartan-3AN & $-3.88000^{*}$ & 0.86952 & 0.000 & -6.164 & -1.596 \\
\hline & Virtex-4 & -0.15500 & 0.86952 & 0.998 & -2.439 & 2.129 \\
\hline & Virtex-5 & $2.74000^{*}$ & 0.86952 & 0.012 & 0.456 & 5.024 \\
\hline \multirow[t]{3}{*}{ Virtex-4 } & Spartan-3AN & $-3.72500 *$ & 0.86952 & 0.000 & -6.009 & -1.441 \\
\hline & Virtex-2 & 0.15500 & 0.86952 & 0.998 & -2.129 & 2.439 \\
\hline & Virtex-5 & $2.89500^{*}$ & 0.86952 & 0.007 & 0.611 & 5.179 \\
\hline \multirow[t]{3}{*}{ Virtex-5 } & Spartan-3AN & $-6.62000^{*}$ & 0.86952 & 0.000 & -8.904 & -4.336 \\
\hline & Virtex-2 & $-2.74000^{*}$ & 0.86952 & 0.012 & -5.024 & -0.456 \\
\hline & Virtex-4 & $-2.89500^{*}$ & 0.86952 & 0.007 & -5.179 & -0.611 \\
\hline
\end{tabular}

*: The mean difference is significant at the 0.05 level 


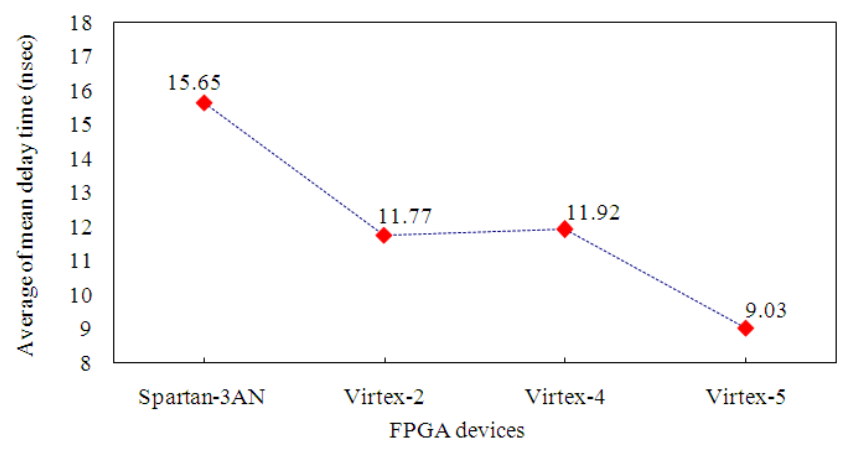

Fig. 1: Average of mean delay time for Spartan-3AN, Virtex-2, Virtex-4 and Virtex-5 for the Braun's multipliers

\section{CONCLUSION}

We have presented hardware design and implementation of FPGA based parallel architecture for standard Braun's multipliers using VHDL. The design was implemented on Xilinx Spartan-3AN XC3S700AN, Virtex-2 XC2V40, Virtex-4 XC4VLX40 and Virtex-5 XC5VLX50 FPGA devices using the ISE $9.2 \mathrm{i}$ design tool. The FPGA resource utilization by Virtex-5 is the lowest in value for $4 \times 4,6 \times 6,8 \times 8$ and $12 \times 12$-bit Braun's multipliers as compared to Spartan$3 \mathrm{AN}$, Virtex-2 and Virtex-4 FPGAs. Even value obtained for Virtex-5 FPGA for $4 \times 4$ bit standard Braun's multiplier for number of occupied slices and look up tables are lower in value than reported in literature. Average connection delays are much lower in Virtex-2 FPGA as compared to Spartan-3AN, Virtex-4 and Virtex-5 FPGAs. As well as there is consistency and gradual increase is seen in Virtex-2 average connection delay. For the maximum pin delay same observation is obtained for Virtex-2 FPGA that the values are gradually increased as the size of multiplier increases. The anomalies in maximum pin delay and average connection delay are observed in Virtex-5, Virtex-4 and Spartan-3AN FPGAs.

There is a statistically significant difference at the 0.05 level in delay time for the four devices compared by using ANOVA and post-hoc Tukey HSD multiple comparison tests at the 0.05 significance level. The test indicates that the mean of delay time for Virtex-5 and Spartan-3AN are significantly different from the other two devices; Virtex-2 and Virtex-4 FPGAs. However, there is no statistically significant difference in mean delay times of the devices between Virtex-2 and Virtex-4.

Spartan-3AN, Virtex-2, Virtex-4 and Virtex-5 FPGA devices also demonstrates that as the size of multipliers increases their mean latency value is also increases.

\section{ACKNOWLEDGEMENT}

The researchers acknowledge the assistance and the financial support provided by the Cornea Research Chair, College of Applied Medical Sciences, King Saud University.

\section{REFERENCES}

Al Mijalli, M.H., 2011a. Spartan-3AN field programmable gate arrays truncated multipliers delay study. Am. J. Applied Sci., 8: 554-557. DOI: 10.3844/ajassp.2011.554.557

Al Mijalli, M.H., 2011b. Delay study of Virtex-2, Virtex-4 and Spartan-3E based truncated multipliers.Int. J. Comp. Sci. and Network Security, 11:7 68-71.

Rais, M.H., 2009a. FPGA design and implementation of fixed width standard and truncated 6×6-bit multipliers: A comparative study. Proceedings of the 4th IEEE International Design and Test Workshop, 15-17 Nov. IEEE Xplore, Riyadh, pp:1-4. DOI: 10.1109/IDT.2009.5404081

Rais, M.H., 2009b. Efficient hardware realization of truncated multipliers using FPGA. World Academy of Science, Engineering and Technology.

Rais, M.H., 2010a. Hardware implementation of truncated multipliers using Spartan 3AN, Virtex-4 and Virtex-5 devices. Am. J. Eng. Applied Sci., 3: 201-206. DOI: 10.3844/ajeassp.2010.201.206

Rais, M.H., 2010b. Hardware design and implementation of fixed width standard and truncated $4 \times 4,6 \times 6,8 \times 8$ and $12 \times 12$ bit multipliers using FPGA. Am. J. Eng. Applied Sci., 3: 201-206.

Rais, M.H., B.M. Al-Harthi, S.I. Al-Askar and F.K. AlHussein, 2010. Design and field programmable gate array implementation of basic building blocks for power-efficient baugh-wooley multipliers. Am. J. Eng. Applied Sci., 3: 307-311. DOI: 10.3844/ajeassp.2010.307.311 
Rais, M.H. and M.H. Al Mijalli, 2011a. FPGA based fixed width $4 \times 4,6 \times 6,8 \times 8$ and $12 \times 12$ bit multipliers using Spartan-3AN. IJCSNSInt. J. Comp. Sci. Netstudy Security, 11: 61-68.

Rais, M.H. and M.H. Al Mijalli, 2011b. Field programmable gate arrays based realization of truncated multipliers. Am. J. Applied Sci., 8: 681 684. DOI: 10.3844/ajassp.2011.681.684

Rais, M.H. and M.H. Al Mijalli, 2011c. Braun's multipliers: Spartan-3AN based design and implementation. J. Comput. Sci., 7: 1629-1632. DOI: 10.3844 jessp.2011.1629.1632

Yeo, K.S. and K. Roy, 2005. Low Voltage, Low Power Subsystems. 1st Edn. McGraw-Hill Professionalm, New York. ISBN: 007143786X 9780071437868

Xilinx, 2009. Spartan-3 FPGA family datasheet, Xilinx. Anitha, R. and V. Bagyaveereswaran, 2011. Comparative study of Braun's Multipliers using FPGA devices. Int. J. Eng. Sci. Tech., 3: 4785-4793. 